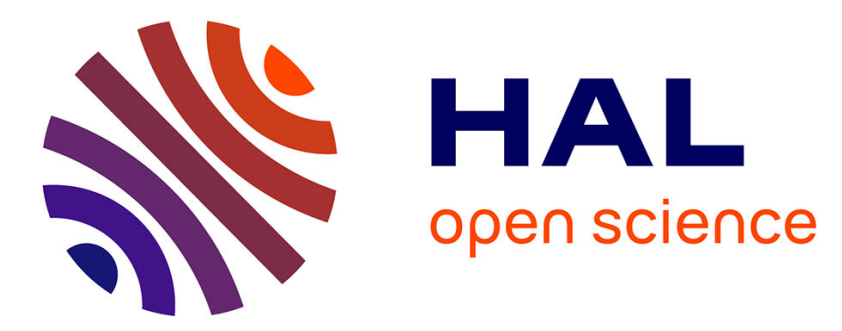

\title{
Comparison of synthetic exposure and sequential exposure for multiple exposure speckle imaging of blood flow
}

\author{
M Chammas, Yu C. H., H. Gurden, Lin H. H., Frédéric Pain
}

\section{- To cite this version:}

M Chammas, Yu C. H., H. Gurden, Lin H. H., Frédéric Pain. Comparison of synthetic exposure and sequential exposure for multiple exposure speckle imaging of blood flow. European Conference on Biomedical Optics, Jun 2021, Munich, Germany. hal-03359533

\section{HAL Id: hal-03359533 https://hal.science/hal-03359533}

Submitted on 30 Sep 2021

HAL is a multi-disciplinary open access archive for the deposit and dissemination of scientific research documents, whether they are published or not. The documents may come from teaching and research institutions in France or abroad, or from public or private research centers.
L'archive ouverte pluridisciplinaire HAL, est destinée au dépôt et à la diffusion de documents scientifiques de niveau recherche, publiés ou non, émanant des établissements d'enseignement et de recherche français ou étrangers, des laboratoires publics ou privés. 


\title{
Comparison of synthetic exposure and sequential exposure for multiple exposure speckle imaging of blood flow
}

\author{
Chammas $\mathrm{M}^{\mathbf{1}}$, Yu $\mathrm{CH}^{2}$, Gurden $\mathrm{H}^{3}$, Lin $\mathrm{HH}^{2}$, Pain, $\mathbf{F}^{\mathbf{1}}$ \\ ${ }^{1}$ Université Paris-Saclay, Institut d'Optique Graduate School, CNRS, Laboratoire Charles Fabry, 91127, Palaiseau, France. \\ ${ }^{2}$ Chang Gung University, Biomedical Imaging Physics Lab, Institute of Radiological Research, Taoyuan City, Taiwan \\ ${ }^{3}$ Université de Paris, CNRS, Laboratoire BFA, 75013, Paris, France \\ marc.chammas@universite-paris-saclay.fr
}

\begin{abstract}
We have compared multiple exposure speckle imaging using two approaches: (i) duration modulation of laser diode pulses with fixed exposure time and (ii) synthetic exposure created from the sum of frames obtained at $1 \mathrm{~ms}$ exposure time. Both methods have been applied to evaluate controlled flows in micro-channels. The results demonstrate that the synthetic exposure method provides accurate speckle contrast data over a wide range of exposures, channel diameters and flows. (C) 2021 The Author(s)
\end{abstract}

\section{Introduction}

Multiple exposure speckle imaging (MESI) has emerged as an efficient and reliable tool for in vivo relative blood flow changes monitoring and tissue perfusion assessment. It has been found as a powerful diagnostic tool in several medical fields such as rheumatology, dermatology, ophthalmology, and neurology among others [1]. MESI has been proven to be more accurate than the previous laser-speckle imaging tools when it comes to flow quantification and the capability of distinguishing the contribution of static scatters (i.e. bones, cartilages, etc.) from that of the moving scatterers (i.e. blood cells) in the speckle pattern. The MESI system developed previously in our lab relied on a posttreatment of speckle images followed by a nonlinear fit to the MESI model in the aim of extracting the functional blood flow maps [2]. However, the acquisition of multiple exposure relied on a fixed camera exposure associated to laser pulses varying in duration and intensity. This implies the use of an acousto-optic modulator (AOM) associated to an arbitrary waveform generator (AWG) and the synchronization of the camera to the laser pulses. We investigate here a much simpler instrumental approach by creating synthetic exposure by the summation of images acquired at a short exposure time (a 10ms synthetic exposure is obtained by the summation of 10 images obtained at $1 \mathrm{~ms}$ ). Such approach has been proposed and validated using high sensitivity, low noise SPAD detectors but at the expense of a limited spatial resolution [3]. Further studies have used CMOS cameras but no comparison to the "classical" AOM was performed as a validation [4,5]. On one hand, the camera noises (dark, read-out and shot noise) add linearly with the exposure time in the synthetic exposure scheme, thus decreasing the speckle contrast. On the other hand, the synthetic exposure scheme is likely to be much less sensitive to the intensity calibration bias observed in the AOM multiple exposure approach. Indeed, in this approach, not only the duration of pulses is modulated, but also their amplitude to keep a similar light level at the detector for all exposure times. The present study was designed to compare the synthetic exposure approach to the laser modulation approach in typical conditions associated with speckle blood flow imaging in the brain of rodents [6].

\section{Materiel and Methods}

The imaging set-up used in this study is showed in figure 1. The laser used is a $634 \mathrm{~nm}$ laser diode (Shangai laser, China) with a maximum output power of $419 \mathrm{~mW}$. A genI sCMOS camera (Orca Flash 2.8, Hamamatsu, Japan) was used. It has a $42 \%$ spectral sensitivity at $634 \mathrm{~nm}$, a typical read noise of $3 \mathrm{e}$ - and a dark noise of 1e-. The microfluidic channels and set-up were described in details previously [2]. Speckle images were recorded for microfluidic channels with a rectangular section of 100,300 and $500 \mu \mathrm{m}$ of widths and $75 \mu \mathrm{m}$ of length. We used $2 \%$ intralipid as the flowing media since its optical scattering properties are close to that of blood. Flows from $1 \mu 1$.min-1 up to $7 \mu 1$.min- 1 were controlled using a pressure controller with inline flow feedback (Fluigent, France).

For all conditions, contrast images were computed using the following equation:

$$
K=\frac{\sigma}{<I>}
$$


Where $\sigma$ is the standard deviation of the intensities in the acquired image, and $\langle I\rangle$ is the average intensity in the same image. A spatial window of $5 \times 5$ is used for the calculation of contrast. The assumption behind the speckle contrast imaging of biological flows is that the flow is inversely proportional to the decorrelation time $\tau_{c}$ of the scatterers. Using multiple exposure contrast images, it is possible to derive $\tau_{c}$ while considering the contribution of static scatterers. Multiple exposure speckle contrast data as a function of exposure are fitted to the following equation [7]:

$$
K(T, \tau c)=\left\{\beta \rho^{2} \frac{e^{-2 x}-1+2 x}{2 x^{2}}+4 \beta \rho(1-\rho) \frac{e^{-x}-1+x}{x^{2}}+v_{\text {noise }}\right\}^{1 / 2}
$$

In the present study, we have acquired and compared multiple exposure speckle contrast data at $T_{\text {exposure }}$ of $1,2,5,10$, 25 and 50ms using two different approaches. In a first approach, called laser modulation method, the acquisition of multiple exposure relies on a camera exposure fixed at $60 \mathrm{~ms}$ associated to laser pulses varying in duration and intensity using an acousto-optic modulator. The width of each pulse is set to the desired exposure duration (same values as the previously chosen 6 exposure times), while its amplitude is set so that the areas for all the pulses (in other words the pulses energies) are equalized. The shortest exposure time requires the maximum amplitude whereas the longest exposure is associated with the lowest amplitude. Contrast images are then calculated for the 6 exposure times using equation (1) and $\tau_{c}$ is derived from a fit of the contrast data to equation (2). In a second approach, called synthetic exposure, contrast images are generated using synthetic exposures as follows: the camera exposure time is set at $1 \mathrm{~ms}$ and the laser diode power is adjusted to ensure that the maximum dynamic of the sensor is used. 50 independent frames at $1 \mathrm{~ms}$ are acquired and contrast images for $T_{\text {exposure }}: 1,2,5,10,25$, and $50 \mathrm{~ms}$ are computed as the sum of the corresponding number of $1 \mathrm{~ms}$-frames. In other words, short exposure frames (1ms) are summed to create longer exposure times. In order to limit the bias due to summation of noise (dark, readout and shot-noise), noise corrections are applied and contrast images are computed using equation (1). Both acquisition set-ups are shown in figure 1.
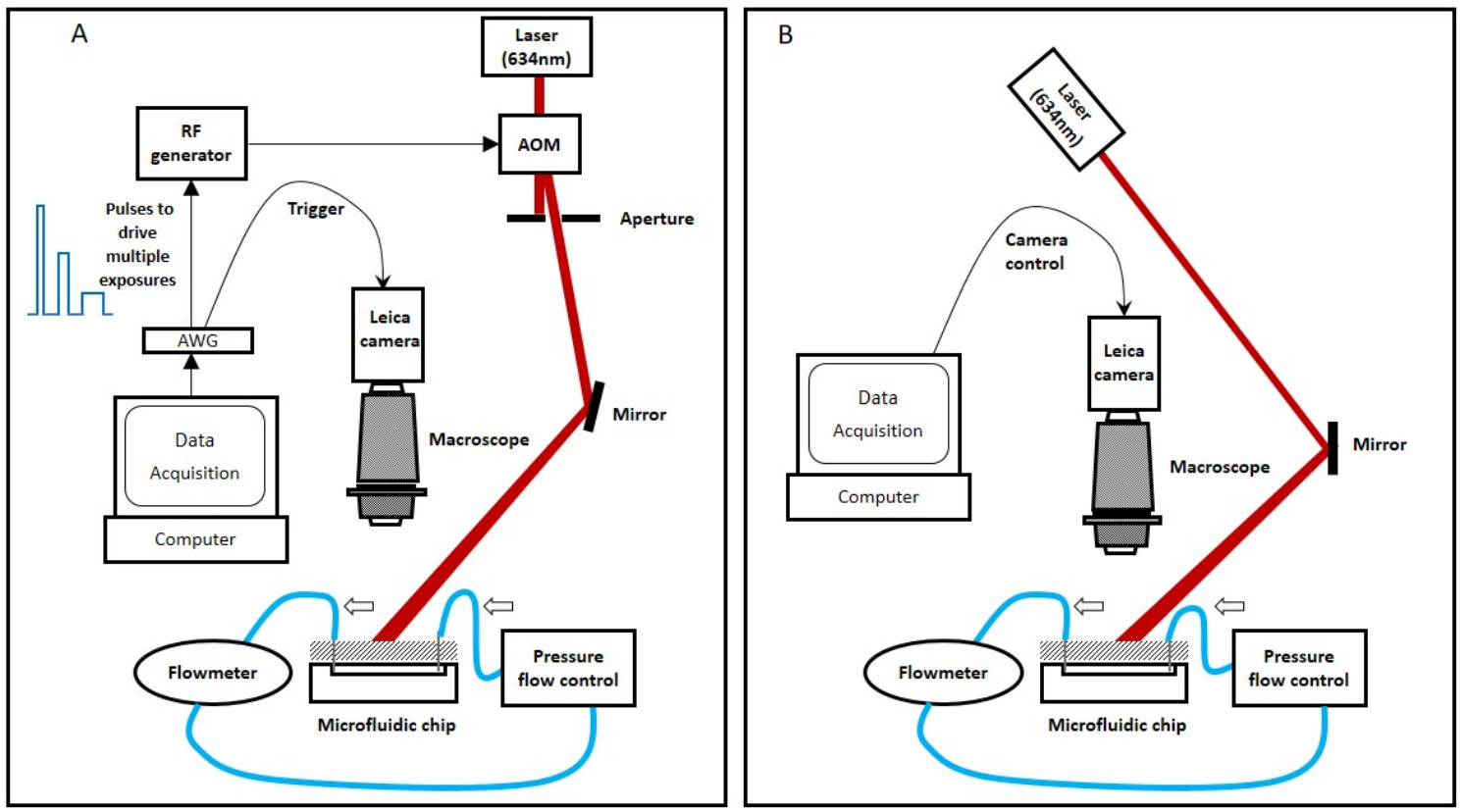

Fig. 1. Set-ups used for laser modulation method (A) and synthetic exposure (B) acquisition of multiple exposure speckle images. 


\section{Preliminary results}
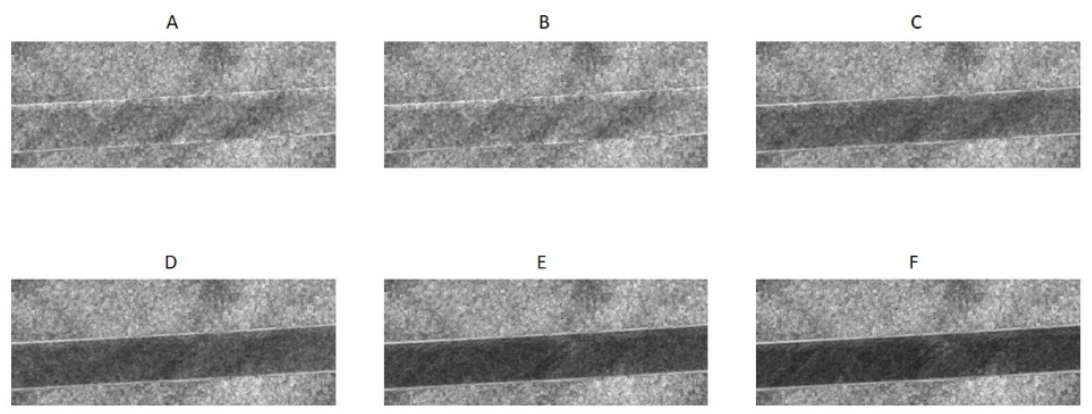

Fig. 2. Speckle contrast images calculated for a channel of $300 \mu \mathrm{m}$ width and a flow of $3 \mu 1 . \mathrm{min}-1$ using the synthetic multi-exposure approach. (A) 1 image acquired at 1ms; (B) 2 images acquired at $1 \mathrm{~ms}$ thus a total exposure of $2 \mathrm{~ms}$; (C) 5 images acquired at $1 \mathrm{~ms}$ thus a total exposure of $5 \mathrm{~ms}$; (D) 10 images acquired at $1 \mathrm{~ms}$ thus a total exposure of $10 \mathrm{~ms}$; (E) 25 images acquired at $1 \mathrm{~ms}$ thus a total exposure of $25 \mathrm{~ms}$; (F) 50 images acquired at $1 \mathrm{~ms}$ thus a total exposure of $50 \mathrm{~ms}$.

\section{References}

1. W. Heeman, W. Steenbergen, G. M. van Dam, and E. C. Boerma, "Clinical applications of laser speckle contrast imaging: a review," J. Biomed. Opt. 24, 1 (2019).

2. H. Soleimanzad, F. Smekens, J. Peyronnet, M. Juchaux, O. Lefebvre, D. Bouville, C. Magnan, H. Gurden, and F. Pain, "Multiple speckle exposure imaging for the study of blood flow changes induced by functional activation of barrel cortex and olfactory bulb in mice," Neurophotonics 6, 015008 (2019).

3. T. Dragojević, J. L. Hollmann, D. Tamborini, D. Portaluppi, M. Buttafava, J. P. Culver, F. Villa, and T. Durduran, "Compact, multiexposure speckle contrast optical spectroscopy (SCOS) device for measuring deep tissue blood flow," Biomed Opt Express 9, 322-334 (2018).

4. S. Sun, B. R. Hayes-Gill, D. He, Y. Zhu, and S. P. Morgan, "Multi-exposure laser speckle contrast imaging using a high frame rate CMOS sensor with a field programmable gate array," Opt Lett 40, 4587-4590 (2015).

5. M. Hultman, I. Fredriksson, M. Larsson, A. Alvandpour, and T. Strömberg, "A 15.6 frames per second 1-megapixel multiple exposure laser speckle contrast imaging setup," J Biophotonics 11, (2018).

6. H. Soleimanzad, M. Montaner, G. Ternier, M. Lemitre, J.-S. Silvestre, N. Kassis, P. Giacobini, C. Magnan, F. Pain, and H. Gurden, "Obesity in Midlife Hampers Resting and Sensory-Evoked Cerebral Blood Flow in Mice," Obesity (Silver Spring) (2020).

7. A. B. Parthasarathy, W. J. Tom, A. Gopal, X. Zhang, and A. K. Dunn, "Robust flow measurement with multi-exposure speckle imaging," Opt Express 16, 1975-1989 (2008). 